Leaflet No. 39.

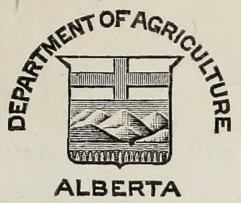

\title{
Production of Quality Market Eggs
}

Published by the direction of

Hon. D. B. MacMillan

Minister of Agriculture
Issued By

POULTRY BRANCH
Distributed by Agricultural Extension Service Edmonton, Alberta.

All too often, eggs are received on the market in a condition that is far from ideal. The quality of the egg is of importance to producers, since the eggs are purchased on a grade basis which takes quality and size into consideration.

Poultrymen can do much to improve the quality of eggs, by sanitary methods of feeding, housing and management of the laying flock, and by proper care of the egg from the time it is laid until it reaches the individual to whom the producer is selling.

\section{THE QUALITY JOURNEY OF AN EGG}

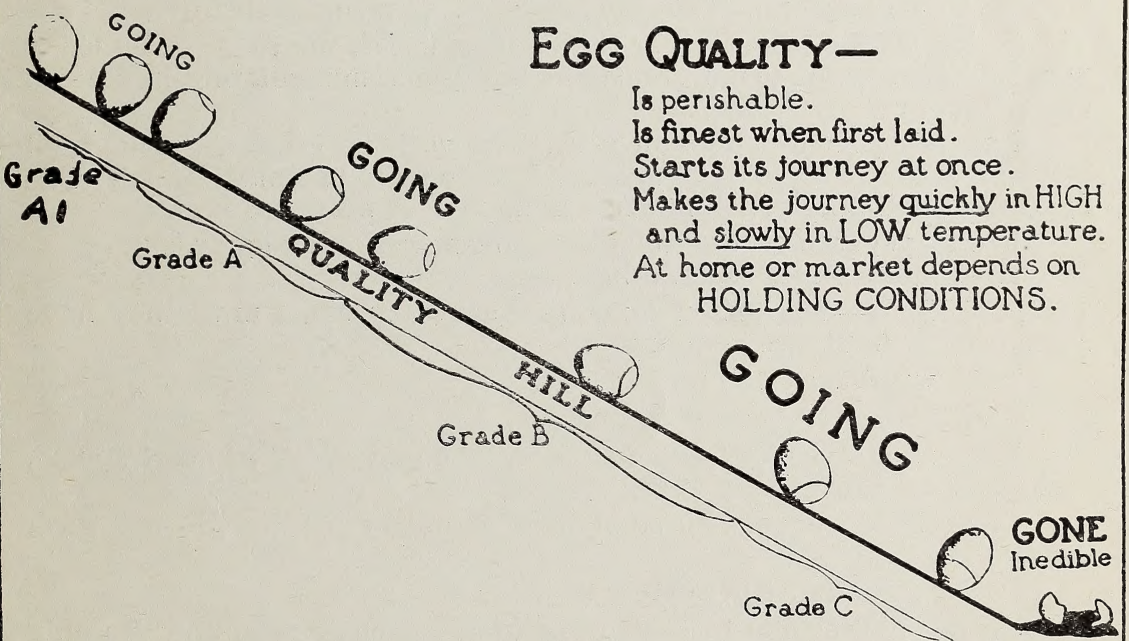

\section{Any Edible Egg is Somewhere on Quality Hill The Grade Tells Where}

Consideration of the following factors affecting egg quality will amply repay any producer.

(1) STOCK-Breed for size, shape and colour of egg. These characters can best be obtained by the use of pedigreed males and carefully selected females, and by setting only eggs of the desired type. 
If purchasing chicks, purchase Alberta Approved chicks from an Alberta Approved Breeder Hatchery or Commercial Hatchery.

(2) MANAGEMENT-Confine layers to the laying house at all times. (Birds allowed to range produce off-coloured yolks and poor flavoured eggs).

Allow at least $3 \frac{1}{2}$ square feet of floor space per bird.

Provide ample litter on the floor and keep reasonably clean and dry.

Clean dropping boards daily.

Provide one nest for each five hens and keep nesting material clean.

Remove male birds promptly after the breeding season.

Provide ample laying mash, oyster shell and grit hopper space and an abundance of clean water. (Plans for hopper and water troughs may be obtained from the Poultry Branch, Department of Agriculture, Edmonton).

(3) FEEDING-Feed according to Government formulae or instructions of commercial feed manufacturers. Laying Mash should contain $1 \%$ Standardized Fish Oil at all times.

Where laying mash contains $5 \%$ Alfalfa Leaf Meal, do not feed other green feed.

Do not allow laying mash, oyster shell, grit hoppers or water troughs to become empty. Keep hoppers and troughs clean.

(4) CARE OF EGGS-Gather the eggs at least four times daily using wire egg baskets or perforated pails and place immediately in a clean egg room or cellar.

Egg room should be well ventilated with no cross draughts, and a temperature as close to 50 degrees $\mathrm{F}$. as possible be maintained. The egg room should be moist, but free from foul or musty odours.

Fruits, vegetables, etc., should not be stored in an egg room.

All egg containers should be clean.

(5) PACKING-Eggs should not be packed in cases until they have cooled for at least twelve hours.

Pack with large end up.

Pack according to size and colour.

Eggs should be candled to remove "blood spots", "meat spots", "cracks" and low quality eggs.

Clean soiled eggs with steel wool or emery brush. Do not wash eggs.

Cases, flats and fillers must be clean.

Do not nail covers to centre partitions of egg cases, and use only four nails in each end.

(6) SHIPPING-Ship not less than twice a week.

Clearly mark all cases with the name and address of shipper and consignee.

Select transportation that gives

(1) Protection from high and low temperatures

(2) Minimum and careful handling of the cases

(3) Prompt delivery to the receivers floor.

Do not ship by truck except on surfaced roads. 